\title{
NEWS-G: Search for Light Dark Matter with a Spherical Proportional Counter
}

\section{P. Knights*}

School of Physics and Astronomy, University of Birmingham, Birmingham, B15 2TT, United

\section{Kingdom}

E-mail: p.r.knights@bham.ac.uk

\section{On behalf of the NEWS-G collaboration}

The NEWS-G collaboration is searching for light dark matter candidates using a novel detector concept, the spherical proportional counter. Access to the mass range from $0.05-10 \mathrm{GeV}$ is enabled by the combination of low energy threshold, light gaseous targets $(\mathrm{H}, \mathrm{He}, \mathrm{Ne})$, and highly radio-pure detector construction. Initial NEWS-G results obtained with SEDINE, a $60 \mathrm{~cm}$ in diameter spherical proportional counter operating at LSM (France), excluded for the first time WIMP-like dark matter candidates down to masses of $0.5 \mathrm{GeV}$. NEWS-G has now constructed a $140 \mathrm{~cm}$ in diameter, spherical proportional counter using $4 \mathrm{~N}$ copper with a $500 \mathrm{~m}$ electroplated inner layer. The detector is currently being commissioned in SNOLAB. The detector will use a read-out technology incorporating resistive materials and multiple anodes, which enable high gain and high-pressure operation. Future NEWS-G detectors which will be fully electroformed underground are also being designed.

\footnotetext{
*** The European Physical Society Conference on High Energy Physics (EPS-HEP2021), ***

*** 26-30 July $2021 * * *$

*** Online conference, jointly organized by Universität Hamburg and the research center DESY ***
}

\footnotetext{
*Speaker.
} 


\section{Introduction}

It is well established from cosmological and astronomical observations that the majority of the Universe's matter content is comprised of cold, non-baryonic Dark Matter (DM). While many direct DM search experiments are dedicated to the search for DM candidates in the $10-1000 \mathrm{GeV}$ mass range, theoretical models, such as asymmetric DM and hidden sectors, have motivated the broadening of direct DM searches to lower masses, below a few $\mathrm{GeV}$, e.g. Refs $[2,3]$. Current experimental results are

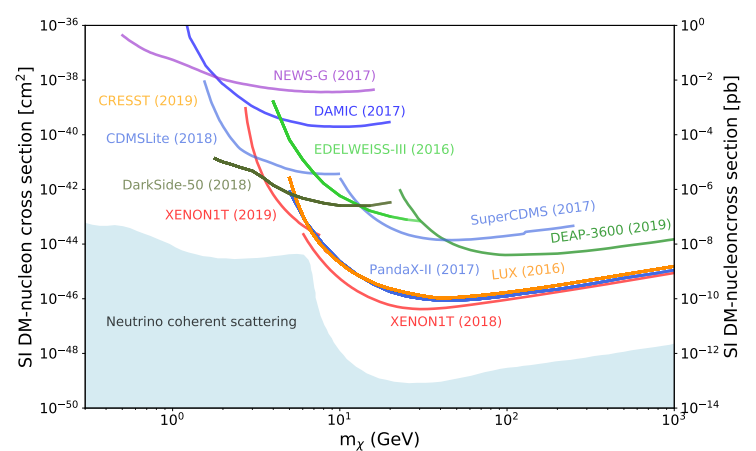

Figure 1: Spin-independent DM-nucleon cross section as a function of DM mass [1]. summarised in Figure 1.

The New Experiments With Spheres-Gas (NEWS-G) collaboration are performing light-DM searches in the range $0.05-10 \mathrm{GeV}$ using a novel gaseous detector, the Spherical Proportional Counter $[4,5,6]$. First results were obtained with a a $\varnothing 60 \mathrm{~cm}$ detector installed in the underground laboratory of Modane (LSM), France. The detector was operated for a total exposure of $9.7 \mathrm{~kg}$. days with a $\mathrm{Ne}: \mathrm{CH}_{4}$ gas mixture and resulted in the first spin-independent (SI) DM-nucleon scattering cross-sections exclusion for a $0.5 \mathrm{GeV}$ mass DM candidate, which was at $4.4 \times 10^{-37} \mathrm{~cm}^{2}$.

Subsequently, NEWS-G has focused on the construction of a $\varnothing 140 \mathrm{~cm}$ detector, and the necessary instrumentation developments for this. The detector, shown in Figure 2, is constructed using $4 \mathrm{~N}$ pure copper with internal $500 \mu \mathrm{m}$ electroplated layer which acts as an internal shield [7]. The detector is installed in SNOLAB, Canada, and is undergoing commissioning, having been first operated in LSM.

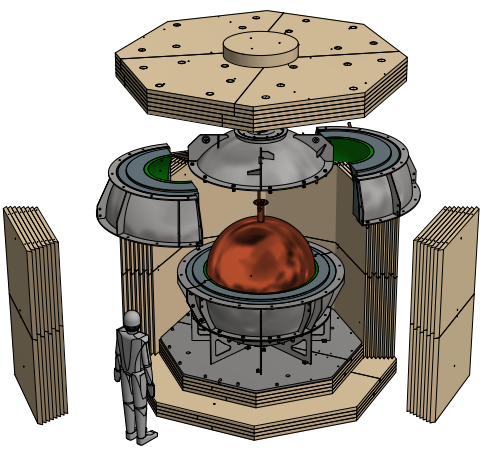

(a)

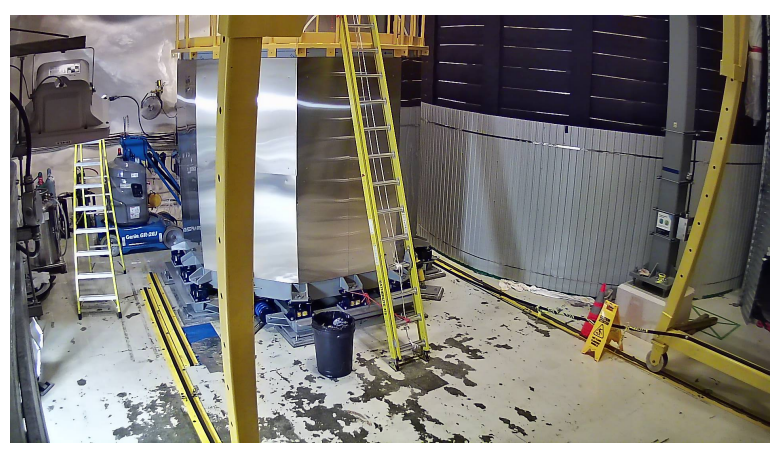

(b)

Figure 2: Schematic (a) and photo (b) of of the $\varnothing 140 \mathrm{~cm}$ NEWS-G detector and shielding comprising $3 \mathrm{~cm}$ archaeological $\mathrm{Pb}, 22 \mathrm{~cm}$ low radioactivity $\mathrm{Pb}$ and $40 \mathrm{~cm}$ high-density polyethylene. 


\section{The Spherical Proportional Counter}

The detector used by NEWS-G is the spherical proportional counter [4, 5, 9, 6], shown in Figure 3. It is composed of an $\mathscr{O}(1 \mathrm{~m})$ diameter grounded spherical cathode with a readout electrode at the centre. The central read-out used is either a $\mathscr{O}(1 \mathrm{~mm})$ diameter spherical anode [6], in the simplest case, or a multi-anode read-out, ACHINOS $[10,11]$. In the ideal case, the electric field in the detector varies as $1 / r^{2}$, dividing the detector into a drift region, and avalanche region; electrons produced by interactions in the gas drift towards the anode, and at around $100 \mu \mathrm{m}$ from the anode, the field becomes sufficiently large for the electrons to create an avalanche, providing charge amplification.

The spherical proportional counter has several inherent

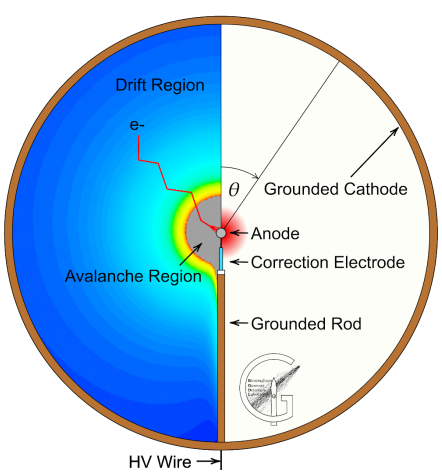

Figure 3: Operational principle of the spherical proportional counter [8]. features which make it well suited for a direct DM search: (1) small number of read-out channels, with future potential for directional sensitivity [11]; (2) detector size-independent capacitance; (3) simple design, with surface-area to volume ratio, and ability to use highly-radiopure materials; (4) stable, high-gain operation; (5) background rejection through pulse-shape analysis; (6) use of light nuclei targets to kinematically match sub-GeV DM particles; and (7) variable operational pressure, gas mixture and voltage provide tools to investigate possible unknown background sources

\section{Read-out Instrumentation}

The simplest read-out mode uses a single spherical anode at the detector's centre. However, in this case, drift and avalanche fields are coupled as both are determined by the anode size and voltage. This limits detector size, operating pressure and stability. ACHINOS, shown in Figure 4a, overcomes this by using multiple anodes at a fixed radius from the detector centre, allowing larger detectors and higher pressure operation. Figure $4 \mathrm{~b}$ shows the increase in electric field at large radii for different read-out configurations

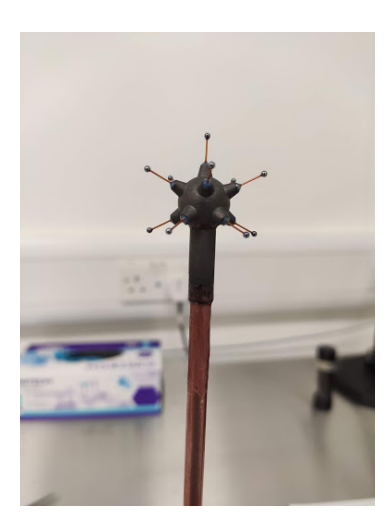

(a)

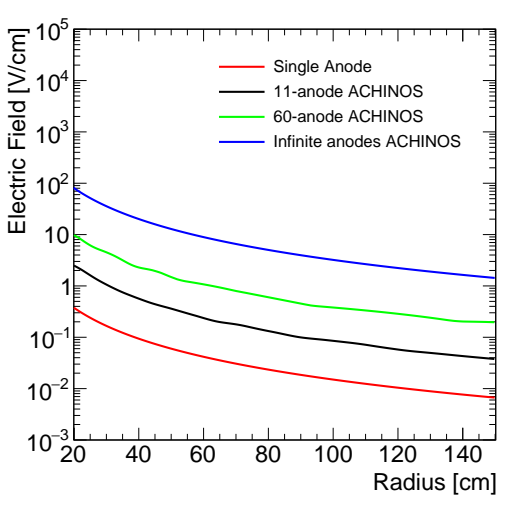

(b)

Figure 4: (a) An 11-anode ACHINOS with DLC-coated 3D printed central electrode. (b) Comparison of electric field magnitude for different read-out configurations. 
Developments in terms of construction precision and operational stability have been achieved using Diamond-Like Carbon (DLC) coated additive manufactured materials. Extensive simulation and experimental testing have been performed to study ACHINOS [11]. The individual read-out of anodes has the potential to allow track reconstruction, which is being studied using a dedicated spherical proportional counter simulation framekwork [8].

\section{High Purity Copper Electroforming}

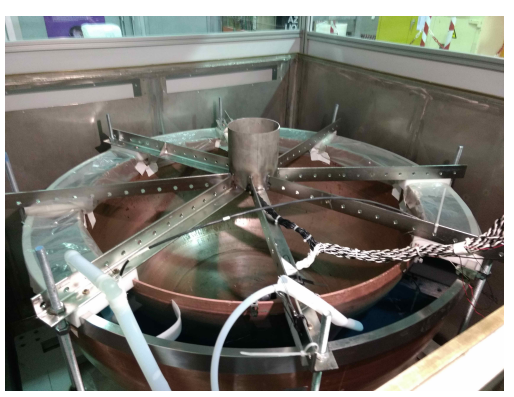

Figure 5: Electroplating set-up showing detector hemisphere, anode, and support structures [7].

During commercial manufacturing processes, ${ }^{222} \mathrm{Rn}$ progeny can be introduced to the bulk of highly pure copper. Alpha spectroscopy assay of $4 \mathrm{~N}$ copper used by NEWS$\mathrm{G}$ indicates a contamination of $29_{-8}^{+8}+9 \mathrm{mBq} \mathrm{kg}^{-1}$ [7]. A method for producing copper with suppressed contamination is potentiostatic electroforming, which exploits the electrochemical properties of copper and contaminant isotopes [12]. This method has been used to produce detector components for several rare event search experiments, e.g. Ref [13].

A $500 \mu \mathrm{m}$ ultra-pure copper layer was electroplated to the inside of the $\varnothing 140 \mathrm{~cm}$ detector to shield it from the underlying $4 \mathrm{~N}$ copper [7]. The set-up for this procedure, which was conducted in LSM, is shown in Figure 5. From Geant 4 simualtions, it is expected that this will significantly reduce the background in the region of interest. The achieved plating rate corresponds to approximately $1.3 \mathrm{~cm} /$ year, which is promising for a future underground fully electroformed sphere.

\section{Future Electroformed Detectors}

The background for the $\varnothing 140 \mathrm{~cm}$ detector is dominated by the natural activity of the $4 \mathrm{~N}$ copper and its cosmogenic activation. Fully electroforming a detector underground in the laboratory where it will be operated would mitigate these contributions. This is the motivation behind the ECuME project, which will establish an electroforming facility underground in SNOLAB, initially for producing a fully electroformed $\varnothing 140 \mathrm{~cm}$ spherical proportional counter. This detector would then be installed in the shielding of the current $\varnothing 140 \mathrm{~cm}$ detector on completion of its physics exploitation. Prototyping for the scale up of current electroforming facilities is currently ongoing, with the construction of the full scale electroformed detector anticipated in 2022. The physics potential of this detector for the DMnucleon SI cross-section is shown in Figure 6.

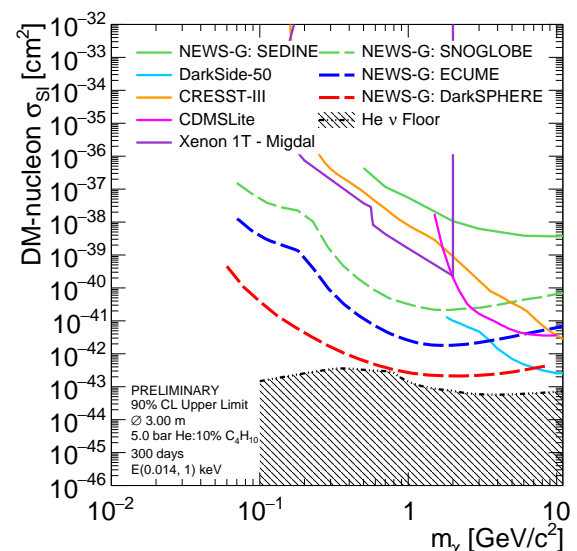

Figure 6: Projected sensitivity of NEWS-G detectors to the spinindependent DM-nucleon crosssection. 
DarkSPHERE, a $\varnothing 300 \mathrm{~cm}$ spherical proportional counter, is being designed for operation in a reduced background, water-based shielding. The Boulby Underground Laboratory, UK, is being targeted as a potential host for DarkSPHERE, with the shielding designed to fit in the Large Experimental Cavern. The reduced background from the shielding, along with the detector being fully electroformed underground, are estimated from Geant 4 simulations to give a total of less than 0.01 counts $/ \mathrm{keV} / \mathrm{kg} /$ day (dru). DarkSPHERE's projected sensitivity to the DM-nucleon SI cross-section reaches the neutrino floor, and is shown in Figure 6.

\section{Summary}

The NEWS-G collaboration are using the spherical proportional counter to probe light DM candidates. Several advances in instrumentation technology, including in ultra-pure materials and in read-out methods, are enabling lower backgrounds, larger detectors, and operation in higher pressures. These developments are promising for future spherical proportional counters which will be fully electroformed underground, and have neutrino-floor reaching potential.

\section{References}

[1] P. Zyla et al., "Review of Particle Physics," PTEP, vol. 2020, no. 8, p. 083C01, 2020.

[2] J. A. Evans, S. Gori, and J. Shelton, "Looking for the WIMP Next Door," JHEP, vol. 02, p. 100, 2018.

[3] K. Petraki and R. R. Volkas, "Review of asymmetric dark matter," Int. J. Mod. Phys. A, vol. 28, p. 1330028, 2013.

[4] I. Giomataris et al., "A Novel large-volume Spherical Detector with Proportional Amplification read-out," J. Instrum., vol. 3, p. P09007, 2008.

[5] I. Savvidis et al., "Low energy recoil detection with a spherical proportional counter," Nucl. Instrum. Meth. A, vol. 877, pp. 220-226, 2018.

[6] I. Katsioulas et al., "A sparkless resistive glass correction electrode for the spherical proportional counter," J. Instrum., vol. 13, no. 11, p. P11006, 2018.

[7] NEWS-G Collaboration, "Copper electroplating for background suppression in the NEWS-G experiment," Nucl. Instrum. Meth. A., p. 164844, 82020.

[8] I. Katsioulas et al., "Development of a simulation framework for spherical proportional counters," $J$. Instrum., vol. 15, no. 06, p. C06013, 2020.

[9] Q. Arnaud et al., "Spherical Proportional Counter: A review of recent developments," J. Phys. Conf. Ser., vol. 1029, no. 1, p. 012006, 2018.

[10] A. Giganon et al., "A multiball read-out for the spherical proportional counter," J. Instrum., vol. 12, no. 12, p. P12031, 2017.

[11] I. Giomataris et al., "A resistive ACHINOS multi-anode structure with DLC coating for spherical proportional counters," J. Instrum., vol. 15, no. 11, p. 11, 2020.

[12] E. W. Hoppe, C. E. Aalseth, O. T. Farmer, T. W. Hossbach, M. Liezers, H. S. Miley, N. R. Overman, and J. H. Reeves, "Reduction of radioactive backgrounds in electroformed copper for ultra-sensitive radiation detectors," Nucl. Instrum. Meth. A, vol. 764, pp. 116-121, 2014.

[13] N. Abgrall et al., "The Majorana Demonstrator Neutrinoless Double-Beta Decay Experiment," Adv. High Energy Phys., vol. 2014, p. 365432, 2014. 